\title{
Modélisation des écoulements induits par la houle sur les plages d'Aquitaine
}

\author{
F. Saint-Cast ${ }^{1}$, B. Castelle $^{2}$, P. Bonneton ${ }^{2}$ et J.P. Caltagirone ${ }^{1}$ \\ ${ }^{I}$ Attaché temporaire d'enseignement et de recherche, Université \\ Bordeaux 1, Laboratoire MASTER-ENSCPB, 16 Avenue Pey Berland, \\ 33607 Pessac Cedex. Email : f.saint-cast@lmaster.u-bordeaux.fr. \\ Tél : 05.56.84.66.81. \\ ${ }^{2}$ Doctorant, Université Bordeaux 1, Département de Géologie et \\ Océanographie, UMR CNRS 5805, Université Bordeaux 1 av. des \\ Facultés, 33405 Talence.Email : b.castelle@epoc.u-bordeaux.fr. Tél : \\ 05.56.84.88.32.
}

\section{Résumé :}

Cet article présente les premières investigations sur l'influence des barres sableuses en croissant sur les courants moyens induits par les vagues. Il est présenté la modélisation bidimensionnelle horizontale retenue pour représenter l'hydrodynamique moyenne intégrée sur la période de la houle. Plusieurs simulations numériques sont réalisées sur une bathymétrie représentative du littoral aquitain, à marée haute, à marée basse, avec une houle frontale et oblique. La focalisation de la houle par la barre et son déferlement sont qualitativement en accord avec les observations faites sur le terrain. De même, les résultats mettent en évidence l'importance du courant de retour en général et la présence de courants sagittaux stables à marée basse, tandis qu'à marée haute, une forte sensibilité du courant à l'incidence de la houle est relevée. De ce fait, l'influence de la hauteur du plan d'eau sur la structure des courants est mise en avant.

\begin{abstract}
:
The influence of crescentic sand bars on mean currents induced by waves is investigated. A depth integrated model is presented to solve horizontally twodimensional problems. Numerical simulations are obtained on a typical bathymetry of the aquitan coast during high and low tide, with a frontal and oblique swell. Computed swell focalisation and breaking zone are in qualitative agreement with our observations. Moreover, on the one hand our results show the presence of stable rip currents during low tide, on the other hand a strong sensitivity of wave incidence to currents is obtained. Thus, the sea level influence over current pattern is revealed.
\end{abstract}

\section{Introduction}

Le littoral aquitain est une côte sableuse non-consolidée soumise aux houles très énergétiques de l'Atlantique Nord. Son étude a permis l'observation de barres en croissant dans la zone subtidale. Leur rôle est essentiel vis-à-vis de l'érosion de la côte, en particulier durant les épisodes de tempêtes où les courants sont violents et les changements morphodynamiques rapides. L'objectif est de 
modéliser ces courants moyens induits par le champ de houle au-dessus de ces barres à partir d'un modèle d'écoulement moyen en 2D intégré sur la verticale. Le mouvement moyen des vagues joue un rôle moteur prépondérant dans la dynamique des courants moyens.

\section{La côte aquitaine}

\subsection{L'hydrodynamique littorale}

La houle au large est de secteur N-NW dominant $75 \%$ de W, 25\% de NW et $5 \%$ de $\mathrm{SW}$ ). La période moyenne est de $\mathrm{T}_{\text {moy }}=8 \mathrm{~s}$, mais elle peut atteindre 14 à 15 secondes (dépressions creuses et lointaines). Son amplitude moyenne est de $\mathrm{H}_{\text {moy }}=1.5 \mathrm{~m}$, cependant elle peut dépasser $6 \mathrm{~m}$ lors des tempêtes décennales.

En arrivant sur la plage, les vagues engendrent des courants moyens particulièrement importants dans la zone de déferlement. Par exemple, lors de la campagne d'octobre 2001 du PNEC-ART7 (Programme National d'Environnement Côtier), pour une houle frontale de hauteur $3 \mathrm{~m}$ et de période $13 \mathrm{~s}$, nous avons relevé des courants de retour (composante transversale du courant moyen dirigée vers le large) de l'ordre de $50 \mathrm{~cm} / \mathrm{s}$. Alors que pour une houle d'incidence $10^{\circ}$, de hauteur $2.5 \mathrm{~m}$ et de période $14 \mathrm{~s}$, les courants de dérive (composante longitudinale) étaient de l'ordre de $1 \mathrm{~m} / \mathrm{s}$ dans la zone de déferlement.

La marée est de type semi-diurne mésotidale (marnage en vives-eaux de $5.5 \mathrm{~m}$ et en mortes-eaux de $2 \mathrm{~m}$ ). Elle engendre des courants rotationnels sur le plateau continental inférieurs à $20 \mathrm{~cm} / \mathrm{s}$. Cependant sur la plage, les courants de marée sont négligeables par rapport aux courants moyens induits par les vagues. Le mouvement de la marée est surtout assimilé à la variation du niveau moyen de la mer.

\subsection{Les plages de sable}

Sur les $230 \mathrm{~km}$ de côte sableuse entre l'estuaire de la Gironde et celui de 1'Adour, on observe des structures sédimentaires périodiques. Dans la zone intertidale, des systèmes de barres et de baïnes sont présents. Dans la zone subtidale, à partir d'images satellite SPOT et de campagnes du Service Hydrographique de la Marine (SHOM), le DGO a également mis en évidence des barres en croissant (Figure 1). Ces barres sont moins mobiles que les barres intertidales, et leur longueur d'onde moyenne est supérieure. Celle-ci varie entre $500 \mathrm{~m}$ et $800 \mathrm{~m}$, mais certains croissants peuvent atteindre $1500 \mathrm{~m}$, [5]. Leur mouvement et leur taille sont étroitement liés aux conditions hydrodynamiques moyennes.

\section{Courant moyen induit par la houle}

\subsection{Modélisation des courants moyens}

L'hypothèse de milieu peu profond permet d'intégrer l'écoulement suivant la verticale entre le fond et la surface libre pour en obtenir une bonne 
représentation bidimensionnelle horizontale $(2 \mathrm{DH})$. Cette hypothèse est justifiée par le fait que l'échelle de longueur horizontale représentative de l'écoulement (longueur d'onde des barres) est très grande devant l'échelle verticale (profondeur). De plus, l'intégration temporelle de l'écoulement sur un période suffisante pour couvrir le spectre de la houle permet de modéliser les tensions de radiation dues au mouvement des vagues $[1,8]$. On donne ci-dessous les équations de conservation de la masse et de la quantité de mouvement du modèle $2 \mathrm{DH}$ dans le repère $\{\vec{x}=(x, y), t\}$ :

$$
\begin{gathered}
\frac{\partial \mathrm{h}}{\partial \mathrm{t}}+\vec{\nabla} \cdot \overrightarrow{\mathrm{Q}}=0 \\
\frac{\partial \overrightarrow{\mathrm{Q}}}{\partial \mathrm{t}}+\vec{\nabla} \cdot\left(\overrightarrow{\mathrm{Q}} \otimes \frac{\overrightarrow{\mathrm{Q}}}{\mathrm{h}}\right)+\mathrm{gh} \vec{\nabla}\left(\mathrm{h}+\mathrm{Z}_{\mathrm{f}}\right)=-\frac{1}{\rho} \vec{\nabla} \cdot \overline{\overline{\mathrm{S}}}+\frac{1}{\rho} \vec{\nabla} \cdot \overline{\overline{\mathrm{T}}}-\frac{\vec{\tau}_{\mathrm{f}}}{\rho}
\end{gathered}
$$

avec $h$ est la hauteur d'eau moyenne, $Z_{f}$ est la cote $d u$ fond, $\rho$ est la masse volumique du fluide et $g$ est l'accélération due à la gravité. $\vec{\tau}_{\mathrm{f}}$ est la contrainte de frottement sur le fond, $\overline{\overline{\mathrm{S}}}$ est le tenseur des contraintes de radiation et $\overline{\overline{\mathrm{T}}}$ représente le tenseur des contraintes visqueuses. Le débit total $\overrightarrow{\mathrm{Q}}$ est défini par :

$$
\overrightarrow{\mathrm{Q}}(\overrightarrow{\mathrm{x}}, \mathrm{t})=\frac{1}{\mathrm{~T}} \int_{\mathrm{t}-\mathrm{T} / 2}^{\mathrm{t}+\mathrm{T} / 2} \int_{\mathrm{z}_{\mathrm{f}}}^{\mathrm{z}_{\mathrm{f}}+\mathrm{h}} \overrightarrow{\mathrm{u}}\left(\overrightarrow{\mathrm{x}}, \mathrm{z}, \mathrm{t}^{\prime}\right) \mathrm{dz} d \mathrm{t}^{\prime}
$$

Or, nous supposons que le champ de vitesse locale $\overrightarrow{\mathrm{u}}(\overrightarrow{\mathrm{x}}, \mathrm{z}, \mathrm{t})$ est constitué d'un courant moyen uniforme sur la verticale $\vec{U}(\vec{x}, t)$ et d'une partie fluctuante déterminée par la houle $\overrightarrow{\widetilde{u}}(\vec{x}, z, t)$ :

$$
\overrightarrow{\mathrm{u}}=\overrightarrow{\mathrm{U}}+\overrightarrow{\widetilde{\mathrm{u}}} \text { où } \frac{1}{\mathrm{~T}} \int_{\mathrm{t}-\mathrm{T} / 2}^{\mathrm{t}+\mathrm{T} / 2} \overrightarrow{\widetilde{\mathrm{u}}}\left(\overrightarrow{\mathrm{x}}, z, \mathrm{t}^{\prime}\right) \mathrm{dt} \mathrm{t}^{\prime}=0
$$

Le débit apporté par les vagues est alors défini par:

$$
\overrightarrow{\widetilde{Q}}(\overrightarrow{\mathrm{x}}, \mathrm{t})=\frac{1}{\mathrm{~T}} \int_{\mathrm{t}-\mathrm{T} / 2}^{\mathrm{t}+\mathrm{T} / 2} \int_{Z_{\mathrm{f}}}^{Z_{\mathrm{f}}+\mathrm{h}} \tilde{\overrightarrow{\mathrm{u}}}\left(\overrightarrow{\mathrm{x}}, \mathrm{z}, \mathrm{t}^{\prime}\right) \mathrm{dzd \textrm {t } ^ { \prime }}
$$

Le courant moyen $\vec{U}=\frac{\vec{Q}-\overrightarrow{\widetilde{Q}}}{h}$ est alors défini par la superposition du courant au sens de Mei $\overrightarrow{\mathrm{U}}_{\text {Mei }}=\frac{\overrightarrow{\mathrm{Q}}}{\mathrm{h}}$ et du courant de compensation par $\overrightarrow{\mathrm{U}}_{\text {Comp }}=-\frac{\overrightarrow{\mathrm{Q}}}{\mathrm{h}}$.

\subsection{Fermeture du modèle}

Nous négligeons les contraintes sur la surface libre, par rapport à la contrainte de cisaillement au fond. Celle-ci est modélisée suivant l'hypothèse que le courant est faible devant la vitesse orbitale des vagues $\left(\vec{U}_{w}\right),[3,6]$ :

$$
\vec{\tau}_{f}=C_{f} \frac{\left\|\vec{U}_{w}\right\|}{\pi} \frac{\vec{Q}}{h} \text { avec } C_{f}=0.0015
$$

Une bonne modélisation de la houle et de son déferlement est essentielle dans l'hydrodynamique côtière, en effet la chute brutale de la hauteur des vagues lors du déferlement se traduit par de forts gradients de tension de radiations. Ces 
contraintes agissent comme une force motrice sur les masses d'eau et influencent de façon déterminante la dynamique des courants moyens. Le champ de vagues et les contraintes de radiations sont déterminées par le modèle de houle REFDIF, [4]. Ce code est basé sur une forme parabolisée de l'équation de Berkoff qui décrit le comportement de la houle linéaire progressive en présence de courant, [7]. Les tensions de radiations ont été programmées dans le modèle de houle selon la formulation de Mei, [8]:

$$
\overline{\bar{S}}=\frac{E}{2}\left[\frac{\overrightarrow{\mathrm{k}} \otimes \overrightarrow{\mathrm{k}}}{\mathrm{k}^{2}}\left(1+\frac{2 \mathrm{kh}}{\sinh (2 \mathrm{kh})}-\frac{\mathrm{Ek}^{2}}{\rho h \omega^{2}}\right)+\overline{\bar{\delta}} \frac{2 \mathrm{kh}}{\sinh (2 \mathrm{kh})}\right]
$$

Nous y avons rajouté un terme du second ordre relatif au débit apporté par les vagues, celui-ci est obtenu en suivant la théorie linéaire, [2] :

$$
\overrightarrow{\widetilde{Q}}=\frac{E}{\rho} \frac{\overrightarrow{\mathrm{k}}}{\omega} \text { où } E=\frac{1}{8} \rho g \mathrm{H}^{2}
$$

avec $E$ l'énergie des vagues, $H$ l'amplitude des vagues, $\mathrm{k}$ le nombre d'onde local et $\omega$ la pulsation de l'onde.

Les contraintes visqueuses sont négligeables devant les contraintes de radiation. Cependant, comme le cisaillement des courants moyens génère des tourbillons, pour les « filtrer » et ne conserver qu'une représentation moyenne des courants, nous introduisons un modèle de mélange latéral. Par analogie avec le tenseur des contraintes de Reynolds, nous supposons que la dissipation est directement exprimée en fonction des variations du courant moyen :

$$
\overline{\bar{T}}=\rho v_{t} h\left([\vec{\nabla} \cdot \vec{U}]+[\vec{\nabla} \cdot \vec{U}]^{T}\right) \text { avec } v_{t}=0.016 \sqrt{g^{2} L_{t}}
$$

où $L_{t}$ représente la distance à la ligne d'eau.

\section{Simulation numérique}

Après avoir défini une bathymétrie type, nous avons simulé les courants moyens, dans le cas d'une houle frontale et pour une houle oblique d'incidence $10^{\circ}$ (hauteur $1.5 \mathrm{~m}$, période $10 \mathrm{~s}$ ), à marée haute puis à marée basse (marnage $5 \mathrm{~m}$ ). Pour limiter l'influence des conditions limites latérales, la houle est calculée sur trois longueurs d'onde, et les courants sont obtenus en périodique sur une seule longueur d'onde.

\subsection{Modélisation de la bathymétrie}

En moyennant différentes caractéristiques (longueur d'onde, pentes, profondeur...), nous avons créé une bathymétrie représentative de celles observées sur la côte aquitaine (figure 1 et figure 2). Cette plage «idéalisée » comprend une barre subtidale en croissant d' $1 \mathrm{~km}$ de longueur d'onde et une zone intertidale lissée (profil de tempête).

\subsection{Validation du modèle de houle}

Afin de vérifier que REFDIF décrit bien le comportement de la houle audessus de notre bathymétrie, nous avons comparé les résultats numériques aux photos aériennes en notre possession. Les résultats montrent que les sorties du modèle sont qualitativement en accord avec les observations. En particulier dans 
le cas d'une houle de tempête, nous observons bien que la houle déferle sur les croissants en dissipant la plus grande partie de son énergie, le déferlement s'arrête ensuite dans les fosses de lévigation avant de reprendre jusqu'à la ligne d'eau (figure 3).

\subsection{Analyse des courants moyens}

Le forçage induit par la houle semble globalement peu sensible à l'incidence de la houle, par contre le niveau du plan d'eau l'influence grandement. En effet, à marée haute le forçage est réparti sur le haut de plage avec une amplification locale au niveau du pied des croissants. A marée basse, ce forçage est discontinu avec d'une part une répartition sur le haut de plage en face de la fosse et une amplification locale à côté du pied du croissant, et d'autre part une zone plus au large, sur le point haut du croissant (figure 4).

A marée basse, on observe des courants sagittaux, les masses d'eau vont s'évacuer plus facilement par la fosse de lévigation qui joue un rôle de chenal. Cette chenalisation favorise les recirculations du courant $\mathrm{U}_{\mathrm{Mei}}$ et les rend assez stables devant le changement d'incidence de la houle (Figure 5a,b). Néanmoins, on relève la présence d'un courant de dérive sur le haut de plage. A marée haute la houle oblique déferle dans la zone intertidale plane et induit un courant de dérive plus homogène mais légèrement oscillant (figure $5 \mathrm{f}$ ). En effet, les recirculations du courant $\mathrm{U}_{\mathrm{Mei}}$ que l'on observe pour une houle frontale (Figure 5e) dégénèrent en oscillations en présence d'une houle oblique.

Pour prendre en compte l'apport de masse par les vagues, et obtenir les courants moyens, on superpose le courant de compensation $U_{\text {Comp }}$ au courant $U_{\text {Mei }}$. On obtient alors un courant de retour présent tout au long de la marée et prédominant lorsque la houle est frontale. De ce fait, on explique l'amplification locale du courant de retour dans les zones où le courant $U_{\text {Mei }}$ est dirigé vers le large. Par exemple, à marée haute on observe trois pics d'intensité du courant moyen (Figure $5 \mathrm{~g}, \mathrm{~h}$ ). De même à marée basse, on observe un pic de courant moyen sur le haute de plage et une amplification du courant sagittal sur le croissant à la sortie de la fosse de lévigation (Figure $5 \mathrm{c}, \mathrm{d}$ ).

Quel que soit le niveau de la marée, les courants moyens pour une houle oblique sont plus intenses que pour une houle frontale, ceci est confirmé par les premiers résultats expérimentaux de la campagne d'octobre 2001 du PNEC.

\section{Conclusion :}

Nous avons établi des premiers résultats numériques concernant la structure et l'ordre de grandeur des courants induits par les vagues au dessus des barres en croissant. En plus de la présence des courants classiquement observés sur des plages planes (courant de retour, courant de dérive), la focalisation de la houle sur les points hauts de la bathymétrie et la présence d'une fosse de lévigation stabilisent les recirculations du courant et créent des courants sagittaux. Ainsi, nos résultats mettent en évidence que l'intensité et la localisation des zones de déferlement détermine la structure des courants : l'hydrodynamique sur des plages complexes dépend fortement de la hauteur du plan d'eau. Il est désormais envisagé de valider ces résultats grâce aux données expérimentales de la 
campagne PNEC-ART7, et de les coupler à une simulation de la morphodynamique des corps sableux, [9].

\section{Références}

[1] Bonneton P. (2001) - Courants moyens générés par la houle en zone de surf. Rapport interne DGO/UMR CNRS 5805.

[2] Castelle B. (2001) - Modélisation de la propagation de la houle et des courants induits au-dessus des barres en croissant : application à la côte aquitaine, DEA de l'université de Marseille, 2001.

[3] James I. D. (1974) - A non-linear theory of longshore currents. Estuar. Coast. Mar. Sci. 2, pp235-249.

[4] Kirby J.T. and R.A., Dalrymple (1994), REF/DIF 1,CACR Report, No. 94-22.

[5] Lafon V., De Melo Apoluceno D., R. Butel, Dupuis H., Michel D., Howa H. et Castelle B. (2001) - Rhytmic Subtidal and Intertidal Bar Morphology and Dynamics in a Mixed-Energy Environment.

[6] Liu P. L. F. et Dalrymple R. A. (1978) - Bottom frictional stresses and longshore currents due to waves with large angles of incidence. Journal of Marine Research, 36, pp357-375

[7] Longuet-Higgins M. S. (1970) - Longshore current generated by obliquely incident sea waves. Journal of Geophysical Research.75, pp6778-6801.

[8] Mei C.C. (1989) The applied dynamics of ocean surface waves. World Scientific.

[9] Saint-Cast F., Bonneton P. et Caltagirone J. P. (2001) - On the splitting of the sediment fluxes balance : a new formulation for the sand waves equation. Proceeding of Coastal Engineering, Grèce.

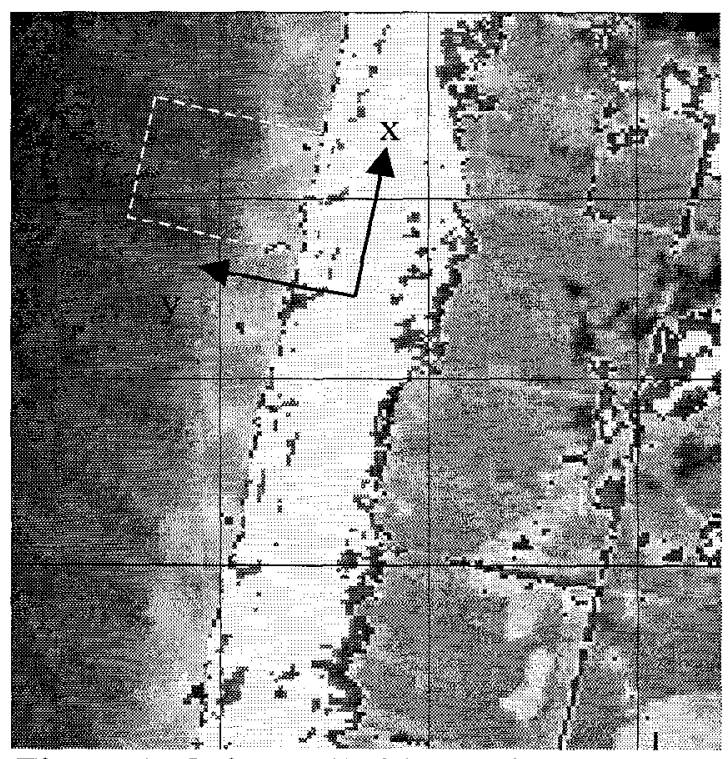

Figure 1 : Mise en Evidence des barres en croissant, Image SPOT, 16/07/1999@

CNES, DGO/UMR 5805 EPOC

Figure 1 : SPOT image showing crescentic bars.

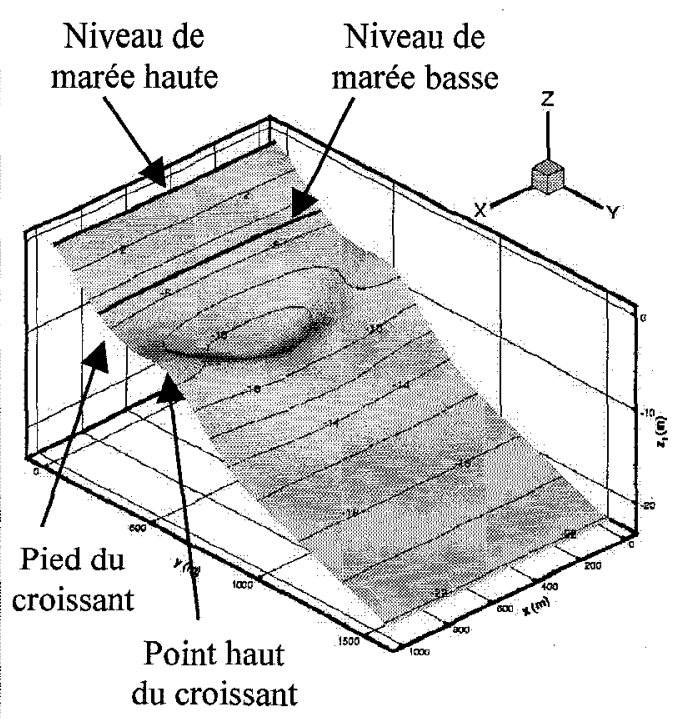

Figure 2 : Représentation 3D de la bathymétrique (une longueur d'onde). Figure $2: 3 D$ view of one wave length of the bathymetry. 

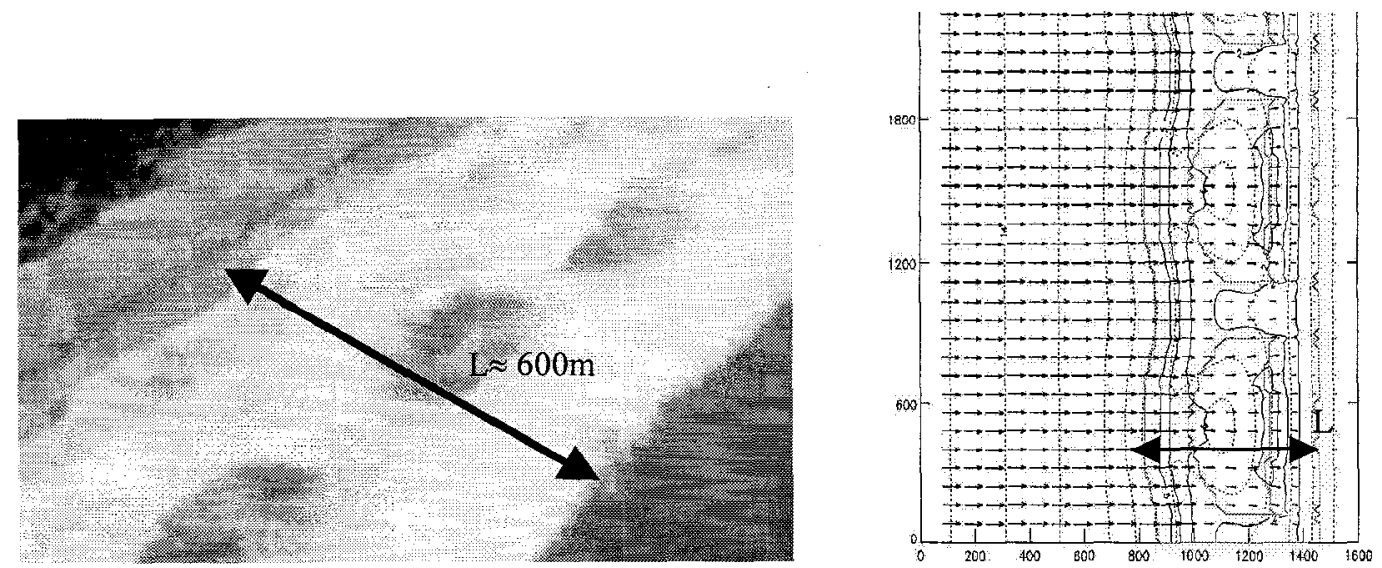

Figure 3 : Comparaison qualitative de la zone de déferlement pour une houle frontale de hauteur $5 \mathrm{~m}$ et de période $13 \mathrm{~s}$.

Figure 3 : Qualitative comparison of the surf zone for frontal waves.
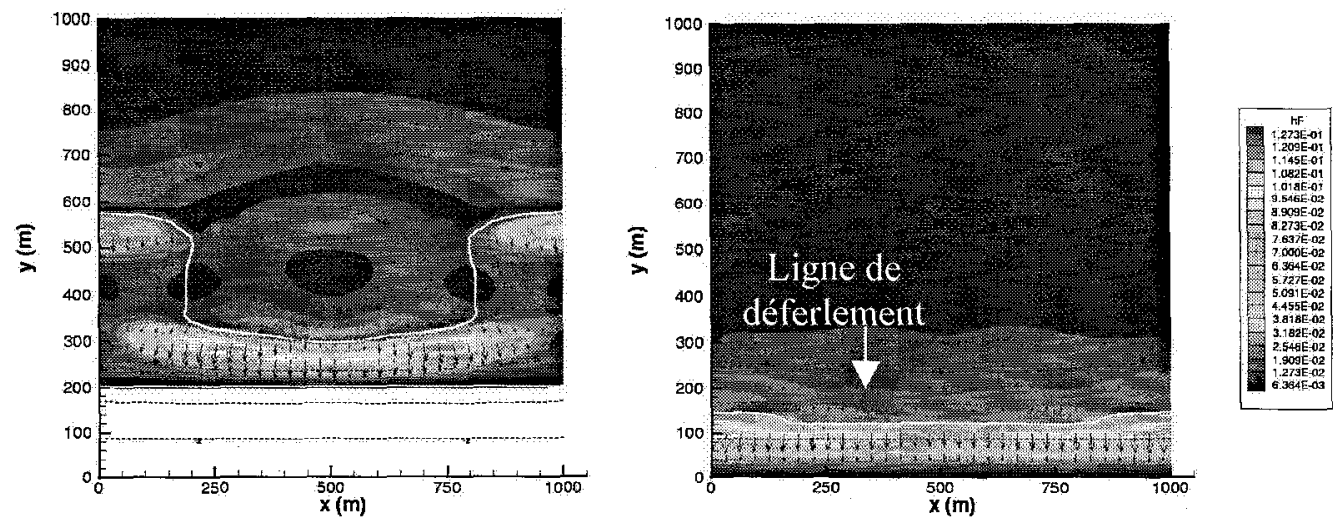

Figure $4:$ Forçage induit par les vagues $h \vec{F}=-\frac{1}{\rho} \vec{\nabla} \cdot \overline{\bar{S}}$.

Figure 4 : Wave induced forcing. 
(a)

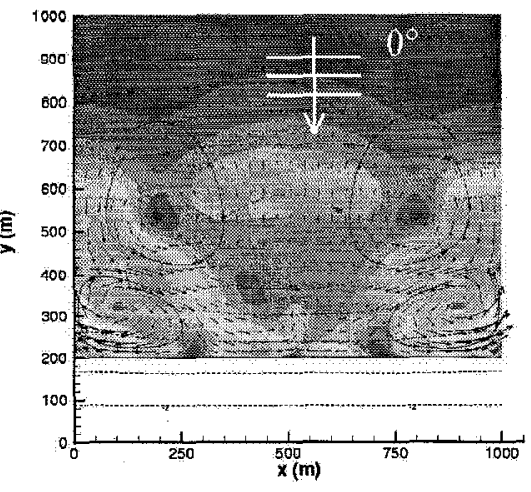

(b)

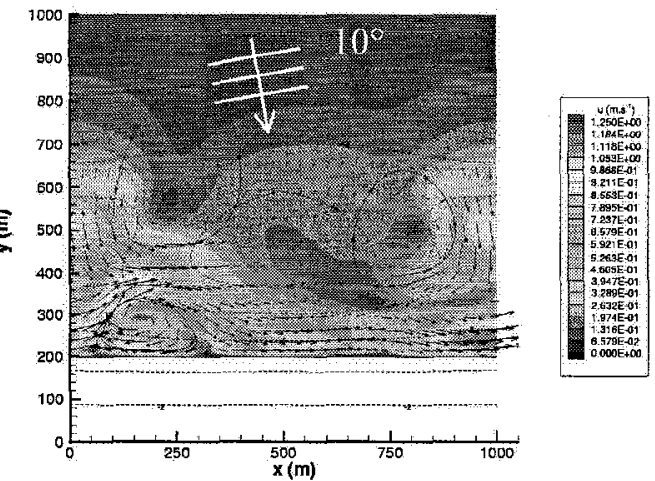

(c)

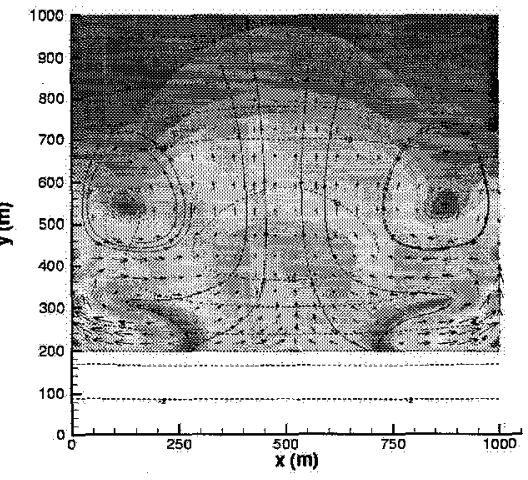

(d)

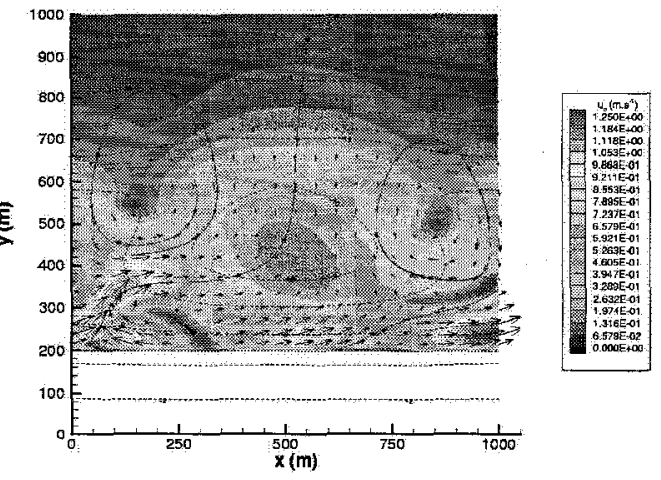

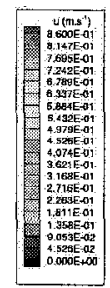

(e)

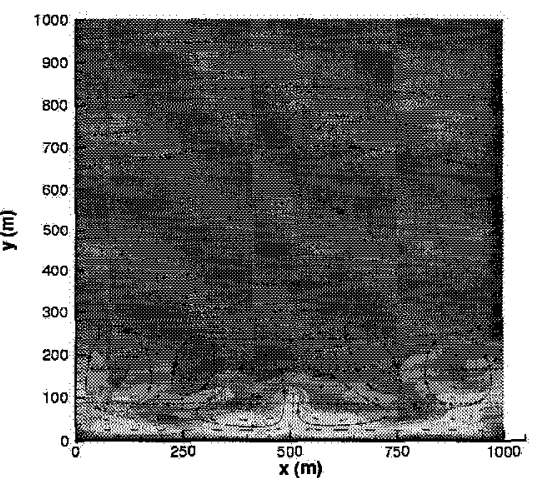

(f)
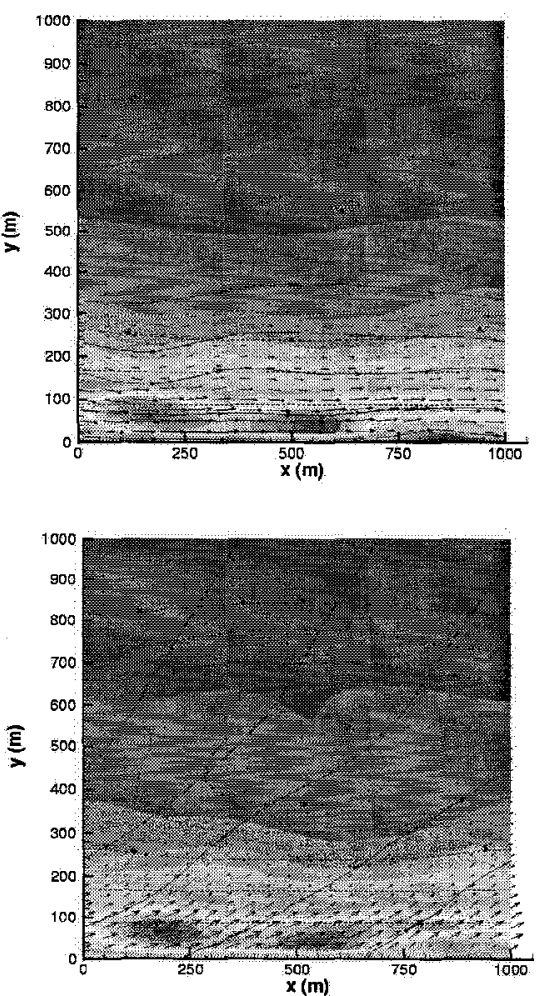

(h)

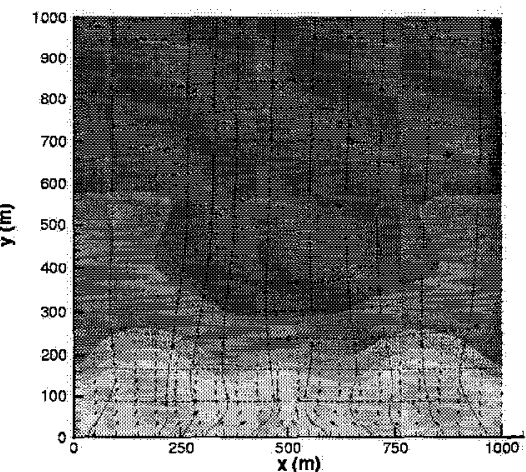

(g)

Figure 5 : Courant moyen (Uc) et courant moyen selon Mei (U), pour une houle de $1.5 \mathrm{~m}$ avec une période de $10 \mathrm{~s}$, à marée basse $(\mathrm{a}, \mathrm{b}, \mathrm{c}, \mathrm{d})$ et à marée haute $(\mathrm{e}, \mathrm{f}, \mathrm{g}, \mathrm{h})$, pour une incidence de $0^{\circ}$ et $10^{\circ}$ (à gauche et à droite).

Figure 5 : Mean current (Uc) and Mean current according to Mei $(U)$. 